\title{
Cambios paisajísticos y efectos medioambientales debidos a la agricultura intensiva en la Comarca de Campo de Cartagena-Mar Menor (Murcia) ${ }^{1}$ Landscape changes and environmental effects due to intensive agriculture in the Campo de Cartagena-Mar Menor (Murcia)
}

\author{
Agustín Caballero Pedraza, Asunción Romero Díaz \\ e Inmaculada Espinosa Soto ${ }^{2}$
}

\section{INTRODUCCIÓN}

1. Los cultivos en invernaderos y su importancia

El cultivo bajo invernaderos no es algo reciente, sino que se remonta a la época romana, en donde las plantas se colocaban bajo estructuras cubiertas con telas impregnadas en aceite, conocidas como «specularia», o con láminas de mica (Tesi, 1972). Los que podrían denominarse invernaderos modernos se elaboraron con vidrio y se construyeron en Italia en el siglo XIII para albergar las plantas exóticas que los exploradores traían de los trópicos. La utilización de los materiales plásticos como cubiertas de invernadero es relativamente reciente, se inició en el año 1948 en EE.UU. con el profesor E. M. Emmert (Uni-

${ }^{1}$ AGRADECIMIENTOS: Los autores quieren agradecer a los revisores anónimos su contribución a la mejora de este trabajo.

2 Departamento de Geografía. Campus de La Merced. Universidad de Murcia, 30.001 Murcia. E-mail: arodi@um.es, acp29398@um.es 
versidad de Kentucky), quien tuvo la idea de sustituir el vidrio por celulosa regenerada (papel celofán) para cubrir una estructura de madera. Desde entonces los invernaderos de plástico se utilizan por todo el mundo y han desplazado al vidrio como material de cerramiento.

La superficie de invernaderos en el mundo a principios del siglo XXI superaba las 450.000 ha (FAO, 2013), con un continuo crecimiento en los últimos años, estimándose en un 20\% anual desde 1980 (Pérez Parra et al., 2002). El $66 \%$ del área de invernaderos corresponde a Asia (China, Japón y Corea), seguida de la cuenca mediterránea con un 30\% (Cobos y López, 1998). En Europa el mayor desarrollo se localiza en los países costeros mediterráneos, debido a las buenas condiciones de insolación y suaves temperaturas invernales (Castilla, 1998). En la actualidad, la superficie total de invernaderos en los países del sur de la Unión Europea es de aproximadamente 90.000 ha, con España a la cabeza (Sigrimis et al., 2009); le siguen Italia, Turquía, Marruecos y Francia (Cotec, 2009).

En España, los primeros invernaderos de plástico se instalaron en 1958 en Canarias y no se extendieron a la Península hasta 1965. Siendo su crecimiento continuo desde entonces (Cotec, 2009). La superficie dedicada a invernaderos, ha aumentado de manera notable en las últimas décadas, se ha pasado de 546 ha en 1968 a 62.065 ha en 2013 (MAAMA, 2013). La mayor concentración de invernaderos se localiza en el sur y sureste peninsular (Andalucía y Murcia) y en Canarias. En el año 2013 los invernaderos en Andalucía representaban el $71 \%$ de la superficie de este sistema de cultivo en España, destacando Almería con una representación del 48,5\% respecto al total nacional. En las Comunidades de Canarias y Murcia los invernaderos ocupan un 11\% en cada una de ellas. El 7\% restante se lo reparten principalmente la Comunidad Valenciana, Cataluña, Navarra y Galicia (MAAMA, 2013).

Si se analiza la última década de 2002 a 2008, la superficie de invernaderos se incrementó en Andalucía y Murcia, pero de 2008 a 2013 se observa una ligera disminución en Andalucía y Canarias; y un descenso más acusado en la Región de Murcia, disminuyendo de 2008 a 2013 en casi 2.500 ha la superficie ocupada por invernaderos (MAAMA, 2002, 2013).

Respecto a la representación de los invernaderos en relación a las tierras de cultivo en 2013, en España sólo representan el 0,4\%, en Andalucía el 1,3\%, pero el $16,6 \%$ en la provincia de Almería; en Canarias el 15,8\% y el 1,4\% en la Región de Murcia.

En todo el sureste de la Península Ibérica, en las últimas décadas, con motivo especialmente del incremento de la agricultura intensiva mediante invernaderos, ha tenido lugar una notable transformación del paisaje. Sin duda es 
de destacar la provincia de Almería (Hernández Porcel, 2005), la cual se ha constituido como modelo paisajístico, tratándose del mayor exponente europeo, y probablemente mundial, de este tipo de agricultura (Tolón Becerra y Lastra Bravo, 2010). Tanto en Europa, como en otros continentes, este modelo de agricultura y, en consecuencia, el desarrollo almeriense, es conocido y envidiado (García Lorca, 1999). Iniciado en los años cincuenta, es a partir de 1977 cuando se comienza a designar el fenómeno como de «milagro almeriense» (García Lorca, 1999). Pero la provincia de Almería no ha sido la única región que ha sufrido una transformación del paisaje a consecuencia de la implantación de invernaderos. Todo el Sureste español se ha caracterizado por un predominio de esta actividad, y este es el caso de la Región de Murcia y de la comarca del Campo de Cartagena-Mar Menor (CCMM) aquí estudiada.

Este tipo de agricultura ha supuesto la clara transformación de una agricultura tradicional de subsistencia a otra tecnificada, en la que se utilizan mejores técnicas y herramientas para lograr el máximo provecho a una determinada superficie. Los efectos económicos y sociales de esta agricultura son positivos, e incluso algunos autores destacan aspectos beneficiosos como pueden ser la mejor utilización de los recursos naturales como el sol, el suelo y, especialmente, el agua (Pérez Parra et al., 2002). No obstante, el impacto paisajístico y ambiental es muy notable (FAUGT, 2008; Gómez, 2011).

La tipología de invernaderos es muy variada. Por las formas y materiales empleados pueden ser invernaderos planos o tipo parral, en túnel o en semicilindro, en capilla a un agua o a dos aguas, de raspa o amagados, asimétricos, etc. Según el material de la cubierta pueden ser de vidrio, de plástico, de plástico y malla (Gil Meseguer y Gómez Espin, 2011). No obstante según Tolón Becerra y Lastra Bravo (2010) se pueden citar dos tipos predominantes: (1) Invernadero tradicional, bajo, cubierto de plásticos y sujetado con postes de madera o aluminio; y (2) Tipo industrial, más alto y de estructura rígida. En la comarca, el invernadero predominante es el tradicional, o «Mediterráneo», propio de las regiones cálidas y se caracteriza por presentar una cubierta de plástico, sin calefacción.

\section{Transformaciones agrarias en la Comarca del Campo de Cartagena-Mar Menor}

Es a principios del S. Xx cuando en la CCMM se produce cierta modernización de la agricultura y un lento inicio del turismo en las riberas del Mar Menor. Estas dos actividades, agricultura y turismo, son la base del actual 
desarrollo comarcal y las que más profundamente han trasformado el paisaje. A mediados del S. xx tuvieron lugar dos hechos importantes con repercusiones para la agricultura de la comarca: (i) El Decreto de 25 de abril de 1993, por el que se reglamentaba la ordenación de los aprovechamientos hidráulicos en la Cuenca del Segura, y por el que se adjudicaba la cantidad de 31 millones de $\mathrm{m}^{3}$ para el riego de cereales del Campo de Cartagena, con caudales excedentes de la Cuenca del Segura; y (ii) la construcción de la central térmica de Escombreras en 1957, con una potencia de 280.000 kW (Espejo Marín 2008), que supuso la electrificación definitiva de la comarca, necesaria para la instalación de motobombas impulsoras de agua del subsuelo. Las aguas subterráneas han contribuido de un modo fundamental al sostenimiento de la actividad agraria de la zona (Gil Meseguer y Gómez Espín, 2014). Pero el trasvase Tajo-Segura ha sido esencial en el cambio de una agricultura tradicional de secano, a una agricultura intensiva de regadío. La llegada de aguas del trasvase Tajo-Segura se produjo a partir del año 1978, si bien con un ritmo muy irregular hasta el año 1995, en que las aportaciones aumentaron considerablemente, hasta alcanzar prácticamente la dotación legal asignada a su zona regable de 122 hm³/año (Martínez Menchón y Senent Alonso 2007). La zona regable de San Pedro del Pinatar, San Javier, Los Alcázares, Torre Pacheco y el Noreste de Cartagena suma una superficie de 27.500 ha, mientras que la parte correspondiente a Fuente Álamo y Murcia tienen una superficie regable de 5.300 ha (González Ortiz, 2007).

La disponibilidad de mayor cantidad de agua, junto a las características climáticas y topográficas de este territorio propició, al igual que en la provincia vecina de Almería, la instalación de invernaderos. Ello ha supuesto una alteración del paisaje tradicional, dando lugar a otro tipo de relaciones e interacciones donde lo tradicional y ecológico ha dado paso a lo económico, con sus ventajas e inconvenientes. Otra actividad que se ha visto afectada ha sido el pastoreo, dificultado por los vallados y los asentamientos de invernaderos, que limitan el movimiento del ganado.

Por otra parte, en la CCMM parece haber una relación entre el crecimiento de la población, la llegada de las aguas del trasvase Tajo-Segura y el desarrollo de la agricultura intensiva de regadío, con los invernaderos como máximo exponente de riqueza económica y de especialización agraria en la comarca. En la figura 1 se puede observar cómo el incremento de la población de los municipios de Los Alcázares, Fuente Álamo, San Javier, San Pedro del Pinatar y Torre Pacheco y la superficie ocupada por invernaderos sigue la misma tendencia interanual, con un crecimiento muy importante, en ambos casos, a partir de 1980. 
FIGURA 1:

POBLACIÓN Y SUPERFICIE DE INVERNADEROS EN LA CCMM

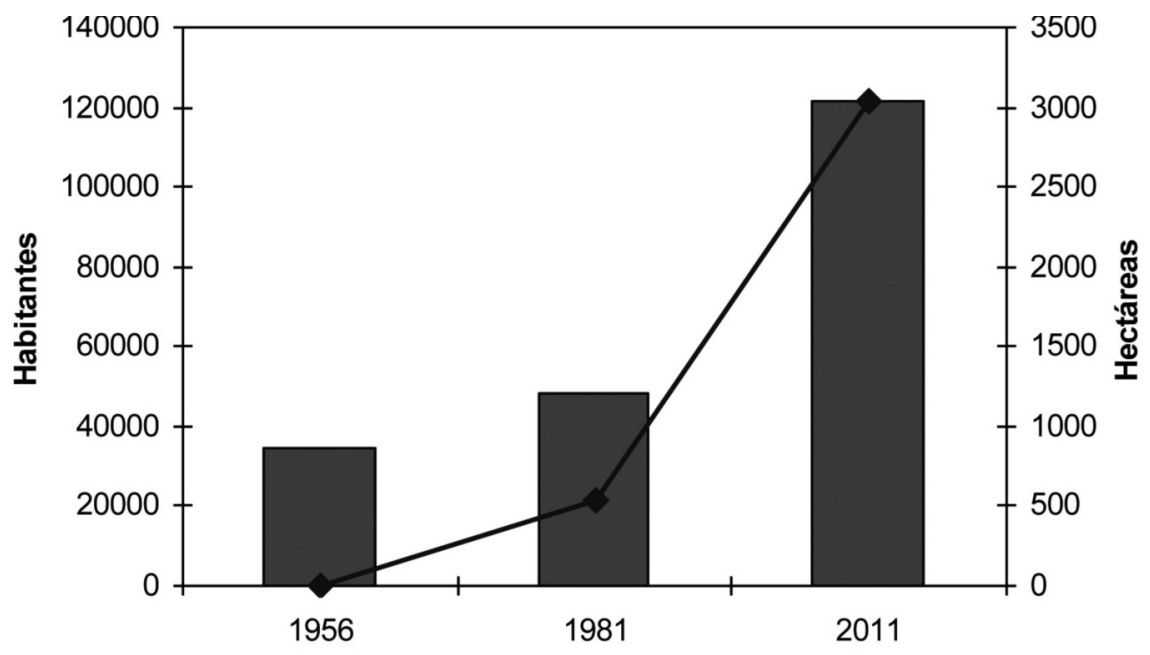

Población $\multimap$ Invernaderos

Fuente: CREM y digitalización propia.

\section{3. Área de estudio}

La comarca del CCMM constituye una amplia planicie vertiente al Mar Menor (albufera cerrada al mar Mediterráneo por la restinga de La Manga), situada en el sureste de la Región de Murcia (figura 2). De acuerdo con la propuesta de comarcas aprobada por el Consejo Regional de Murcia en 1980, en la comarca del Mar Menor estarían incluidos los municipios de Los Alcázares, San Javier, San Pedro del Pinatar y Torre Pacheco, aunque para este estudio se han incluido también los sectores orientales de los municipios de Fuente Álamo y Cartagena, y la parte sur del municipio de Murcia. Este espacio tiene una extensión de 30.574 hectáreas y una población de 107.088 habitantes (INE, 2012).

Desde un punto de vista geológico, la comarca se inscribe en el ámbito de las Cordilleras Béticas y sufrió un hundimiento tectónico que afectó a los estratos paleozoicos depositados y una elevación de los márgenes externos, quedando así enmarcada por una orla de sierras de mediana altitud. El clima de este espacio se caracteriza por recibir escasas precipitaciones (en torno a 300 mm anuales), con una alta irregularidad y, en ocasiones, de alta intensidad. 
Sus temperaturas son suaves en invierno y elevadas en verano, con una media anual de $17^{\circ} \mathrm{C}$. Las escasas precipitaciones, junto a las elevadas temperaturas, determinan que las necesidades de agua sean muy altas y el déficit hídrico supere los $500 \mathrm{~mm}$ al año. No obstante, el elevado número de horas de sol (en torno a 3.000 al año), favorece una agricultura fuera de estación e intensiva (Romero Díaz y Belmonte Serrato, 2011a).

\section{FIGURA 2:}

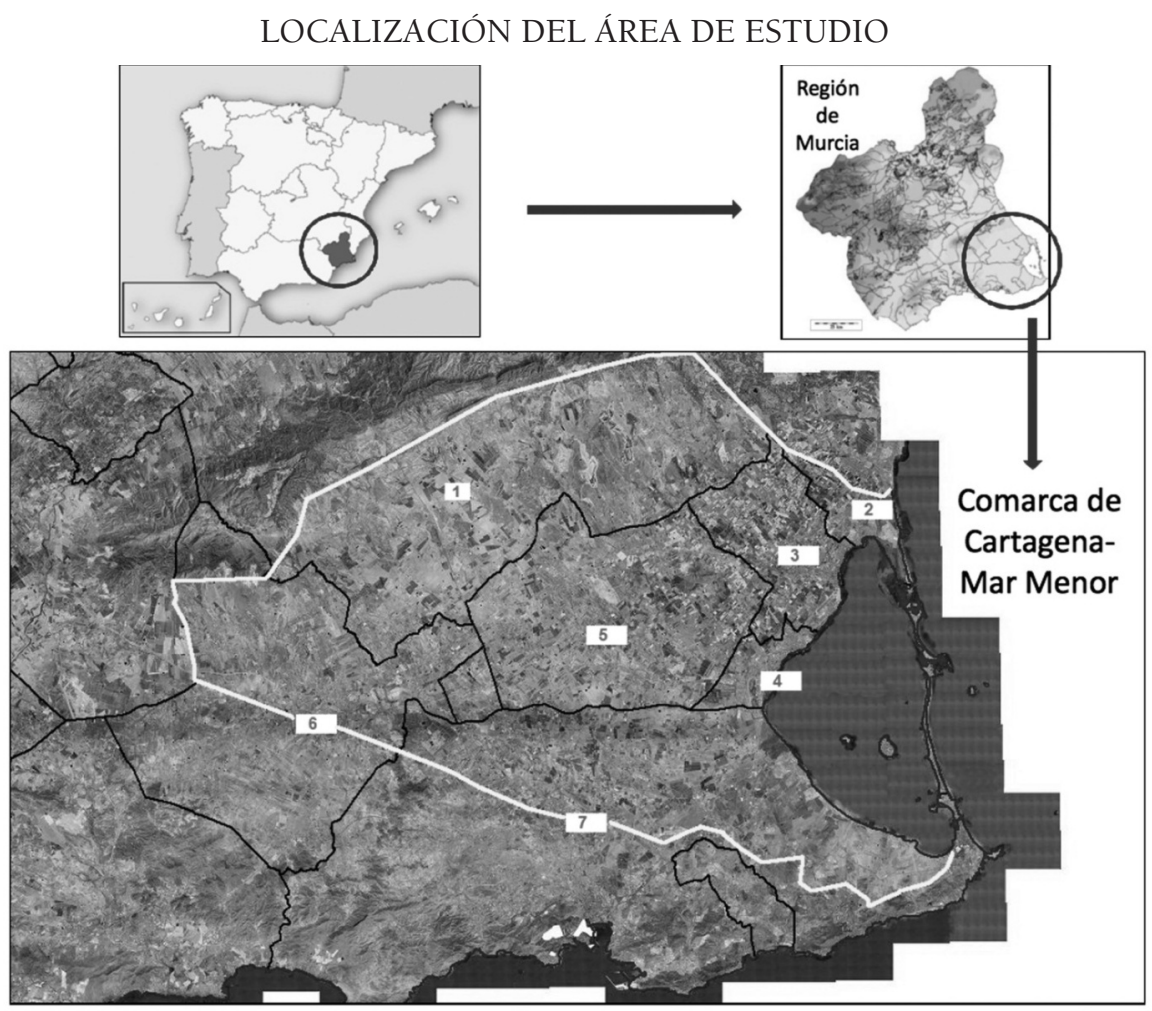

Área de estudio

Municipios de: 1. Murcia, 2. San Pedro del Pinatar, 3. San Javier, 4. Los Alcazares, 5. Torre Pacheco, 6. Fuente Álamo y 7. Cartagena. Fuente: Elaboración propia.

La aridez de la comarca y la escasez de cursos superficiales de agua (ramblas secas, salvo en los escasos días de lluvias intensas) (Conesa García, 1990), reduce mucho las posibilidades de actividad agraria. No obstante, el hombre ha puesto en cultivo grandes superficies de tierras que poseen buenas condiciones 
térmicas y edáficas para la agricultura, si cuentan con el agua suficiente. Desde antiguo, se han extraído aguas subterráneas mediante artilugios tradicionales primero (norias, molinos de viento) y motores eléctricos después (Román Cervantes, 2007), hasta el punto de llegar a la sobreexplotación de los acuíferos. La llegada a esta área de las aguas del Trasvase ha provocado, no sólo la reducción del $50 \%$ de las extracciones de agua subterránea, sino también la recuperación (en algunos acuíferos) de los niveles piezométricos por los excedentes de regadío, debido al retorno de las aguas de riego (Martínez Menchón y Senent Alonso, 2007), incluso en áreas no regadas por el Trasvase.

Los paisajes tradicionales de secano, se han transformado en regadíos de cultivos herbáceos y frutales (especialmente cítricos) y, además, han aparecido otros elementos, los invernaderos, que han contribuido a un profundo cambio paisajístico.

\section{Objetivos}

El objetivo general de este estudio ha sido analizar la transformación del paisaje que, en la comarca del CCMM, ha tenido lugar en las últimas décadas (30 años) como consecuencia de la instalación e incremento de los cultivos bajo invernaderos.

Otros objetivos más específicos han sido: (1) evaluar las superficies de invernaderos en años de referencia (1981 y 2011) en cada uno de los municipios estudiados, mediante digitalización de la superficie dedicada a invernaderos en las ortofotos; (2) evaluar igualmente estas superficies mediante datos estadísticos oficiales; (3) realizar una cartografía de las áreas cubiertas por invernaderos en los distintos años; y (4) analizar algunos de los posibles efectos medioambientales que este tipo de agricultura lleva consigo.

\section{FUENTES Y MÉTODOS}

Se han utilizado ortofotos obtenidas de Cartomur, el Portal Digital del Servicio de Cartografía de la Dirección General de Ordenación del Territorio de la Región de Murcia; comparado distintas ortofotos del período 1970-2011 y digitalizado los límites a través de sistemas de tratamiento de Información Geográfica (SIG), mediante el programa gvSIG; realizado una comparación de datos estadísticos de las últimas décadas a través de los anuarios estadísticos de la Región de Murcia (CREM) y realizado trabajos de campo de comprobación directa para verificar lo observado mediante digitalización. 
RESUltados y DiscusióN

\section{Evolución de la agricultura en la Comarca}

La Comarca del CCMM tiene una gran tradición agrícola y se ha ido modificando y tecnificando con el paso de los años. Se ha producido una especialización agraria, lo que ha dado lugar a una transformación progresiva del paisaje tradicional de secano, con escasos cultivos de regadío tradicional, hacia un paisaje, donde cada vez abundan más los cultivos intensivos bajo plásticos. Se ha producido un cambio radical, que ha afectado no sólo a la distribución de los cultivos y al poblamiento, sino también a la estructura de propiedad de la tierra, los regímenes de tenencia y las características de las explotaciones (Morales Gil, 1997).

Al igual que ha sucedido en otras áreas del sureste español, como es el caso de la provincia de Almería, la concentración de producción de hortalizas en invernadero ha sido resultado de la confluencia de una serie de factores, que pueden agruparse en: recursos naturales, tecnología, capital humano y factor industrial (Tolón Becerra y Lastra Bravo, 2010).

En la actualidad, en la CCMM la superficie ocupada por cultivos de regadío ha aumentado. En 1985 el regadío ocupaba un 38\% frente a un 62\% del secano; por el contrario en 2011 el regadío ocupa el 46,6\% y el secano el $53,4 \%$. No obstante, también hay que mencionar que las tierras de cultivo han disminuido habiendo pasado de 143.793 ha en 1985 a 115.743 ha en 2011 (MAAMA, 2002, 2013).

La segunda mitad de la década de los años ochenta del siglo xx, estuvo marcada por un importante incremento del regadío en la comarca, en detrimento del secano. Desde el año 1985 (55.024 ha) hasta 2002 (75.141 ha), la tendencia de crecimiento fue continua, pero desde el año 2004 la superficie de regadío experimentó un notable descenso; así en el año 2011 la superficie regada en la comarca era de 53.999 ha. Muy posiblemente la disminución de la superficie de cultivo (tanto en secano como en regadío) en la primera década el siglo XXI, puede ser atribuida al aumento de las superficies artificiales (OSE, 2006) y al abandono de campos de cultivos (Martínez Hernández et al., 2013).

A nivel municipal, Cartagena, San Javier, San Pedro del Pinatar y Torre Pacheco tienen la misma tendencia que la comarca; Murcia presenta un descenso continuado en todo el periodo; y Fuente Álamo muestra la tendencia contraria a Murcia, pasando de las 1.228 ha en 1985 a 4.631 en 2011 (figura 3). 
FIGURA 3:

EVOLUCIÓN DE LA SUPERFICIE DE REGADÍO EN LA CCMM

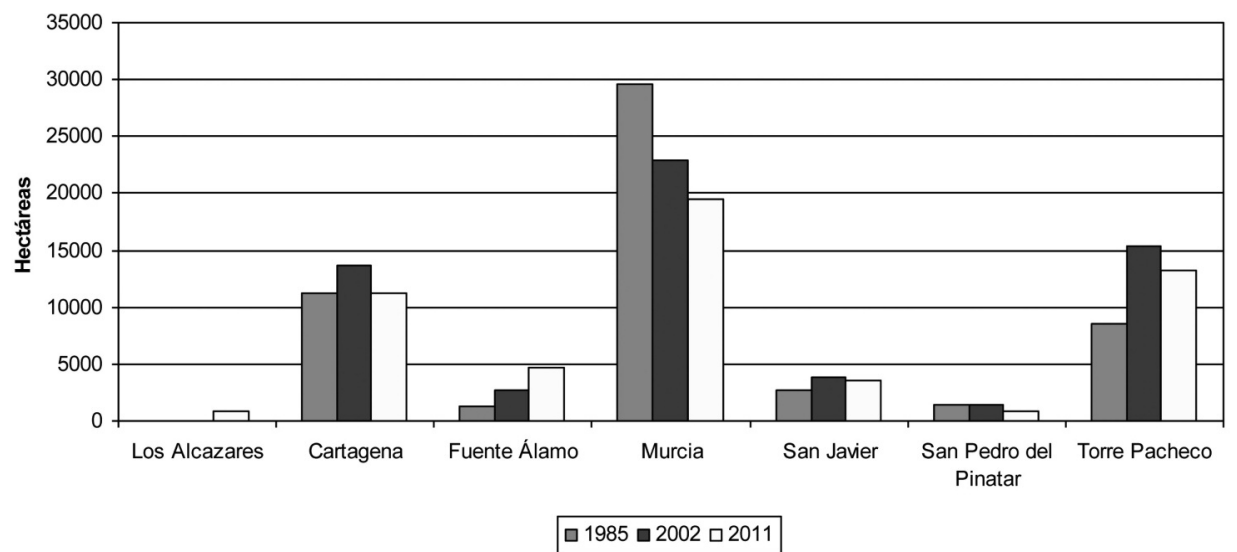

Fuente: Elaboración propia a partir de datos del CREM.

\section{Superficie de invernaderos en la Comarca del Campo de Cartagena-Mar Menor según datos estadísticos}

De la superficie dedicada a regadío, las estadísticas oficiales contabilizan, para el año 2011, 1.495 ha ocupadas por invernaderos, pero estos datos no concuerdan con los valores obtenidos por nosotros mediante digitalización, para el mismo año. En la tabla 1 se muestran las diferencias de superficie de invernaderos, según los datos estadísticos oficiales y las superficies digitalizadas para cada uno de los municipios estudiados.

Es de reseñar cómo la superficie de invernaderos digitalizada es más del doble (3.031 ha) de lo registrado oficialmente, pero cabe señalar que la implantación del invernadero no solo implica la propia instalación específica, sino también instalaciones adyacentes para su correcto funcionamiento, además de la utilización del terreno circundante entre invernaderos; por lo que en el trabajo de digitalización se han contabilizado estas superficies teniendo en cuenta que, en agricultura y economía agraria, el rendimiento agrícola es la producción dividida entre la superficie ( $\mathrm{Tm} / \mathrm{ha}$ ). No obstante, hay que mencionar que en la digitalización se pueden cometer errores, como por ejemplo contabilizar invernaderos que no están en producción, aunque en la imagen se observen en buen estado de conservación. 
TABLA 1:

SUPERFICIE (HA) DE CULTIVOS EN INVERNADEROS EN LOS DISTINTOS MUNICIPIOS ESTUDIADOS. AÑO 2011

\begin{tabular}{lccccc}
\hline & $\begin{array}{c}\text { Superficie } \\
\text { regadío } \\
\text { (CREM) } \\
\text { (ha) }\end{array}$ & $\begin{array}{c}\text { Superficie } \\
\text { invernaderos } \\
\text { (CREM) } \\
\text { (ha) }\end{array}$ & $\begin{array}{c}\text { Invernaderos } \\
\text { respecto } \\
\text { al regadío } \\
\text { (ha) }\end{array}$ & $\begin{array}{c}\text { Superficie } \\
\text { invernaderos } \\
\text { digitalizada } \\
\text { (ha) }\end{array}$ & $\begin{array}{c}\text { Invernaderos } \\
\text { respecto } \\
\text { al regadío } \\
\text { (\%) }\end{array}$ \\
\hline Los Alcázares & 793 & 15 & 1,89 & 19,05 & 2,40 \\
Cartagena & 11.262 & 187 & 1,66 & 244,38 & 2,17 \\
Fuente Álamo & 4.631 & 64 & 0,38 & 107,22 & 2,32 \\
San Javier & 3.521 & 439 & 12,47 & 1002,59 & 28,47 \\
San Pedro P. & 822 & 120 & 14,60 & 199,46 & 24,27 \\
Murcia & 19.429 & 12 & 0,06 & 372,2 & 1,92 \\
T. Pacheco & 13.219 & 658 & 4,98 & 1086,91 & 8,22 \\
\hline Total & 53.677 & 1.495 & 2,76 & 3031,81 & 5,61 \\
\hline
\end{tabular}

Fuente: Elaboración propia a partir de datos del CREM y digitalización propia.

En cuanto a la superficie que los invernaderos representan respecto a la superficie regada, los municipios de San Javier y San Pedro del Pinatar según los datos digitalizados representan el $28,5 \%$ y el $24,3 \%$ de la superficie respectivamente.

Sin duda, la crisis económica actual ha afectado también a la agricultura regional y comarcal, como se observa en la disminución de la superficie ocupada por invernaderos. El caro mantenimiento que necesitan estas instalaciones hizo que muchos de ellos se dejasen inactivos o se destruyeran, es decir, las tierras se dejaron en barbecho o directamente se dejó de cultivar en ellos.

A nivel municipal (figura 4), según datos oficiales, sobresale Torre-Pacheco, seguido de San Javier, como los municipios con mayor superficie de invernaderos. En el caso de Torre-Pacheco la mayor superficie ocupada se dio en la segunda mitad de la década de los noventa del siglo xx, mientras que en San Pedro del Pinatar tuvo lugar en la primera década del siglo xxI, al igual que ocurrió en Cartagena y Fuente Álamo, aunque con menor extensión. 
FIGURA 4:

EVOLUCIÓN DE LA SUPERFICIE DE INVERNADEROS POR MUNICIPIOS

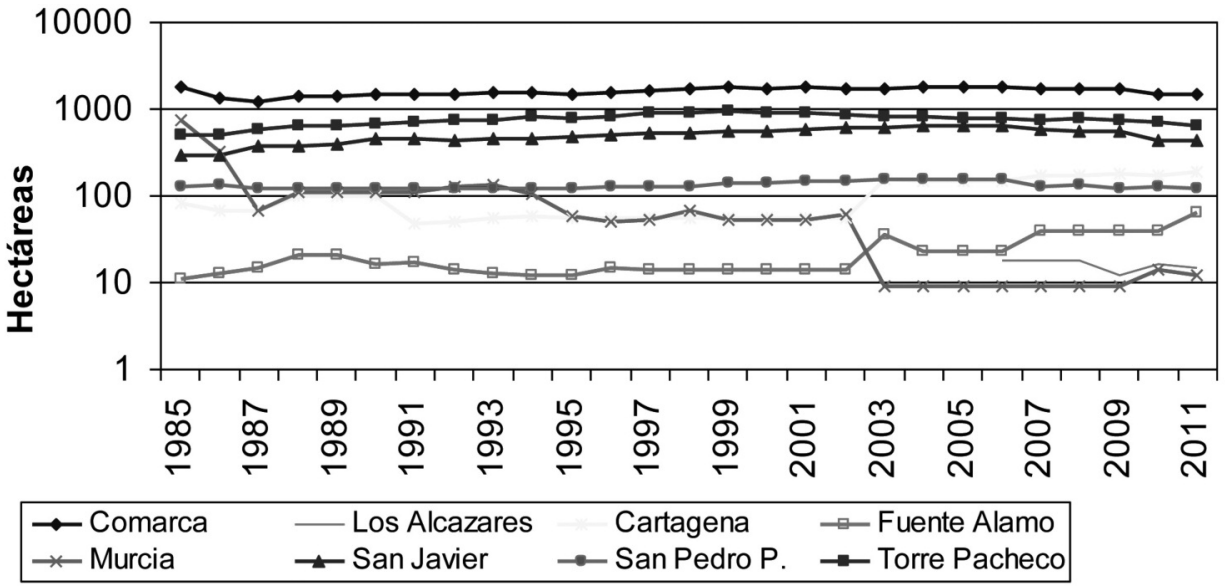

Fuente: Elaboración propia a partir de datos del CREM

\section{Superficie de invernaderos en la Comarca del Campo de Cartagena-Mar Menor según digitalización}

Al analizar las fotografías aéreas de 1956, se observa cómo en este año no hay evidencias de invernaderos. Esto se debe a que este tipo de cultivo, no se había desarrollado suficientemente y requería de aportes hídricos para riego de los que la comarca carecía. Los primeros invernaderos en la comarca (Costa Mas y Canales Martínez, 1988), se instalaron en la campaña 1970-71 en San Pedro del Pinar y en el municipio vecino alicantino del Pilar de la Horadada; con posterioridad se difundieron por la Ribera del Mar Menor y Torre Pacheco. La época en que el ritmo de construcción adquirió mayor rapidez tuvo lugar entre los años 1974 y 1977. En la ortofoto de 1981 ya se observa un número elevado de invernaderos, y es a partir de esta fecha cuando se produce un incremento muy notable hasta la actualidad (figura 1).

Es de reseñar, a nivel municipal (figura 5), el aumento tan significativo que entre los años 1981 y 2011 se produce en la superficie ocupada por invernaderos. A nivel comarcal se pasa de 535 ha a 3.032 ha; y por municipios son Torre Pacheco y San Javier los que han visto aumentar, de forma espectacular, la superficie dedicada a este sistema de cultivo. En el caso de Torre Pacheco en 1981 sólo tenía 127,8 ha de invernaderos y en 2011 superaba las 1.000 ha de superficie. 
FIGURA 5:

SUPERFICIE DE INVERNADEROS POR MUNICIPIOS EN LA CCMM

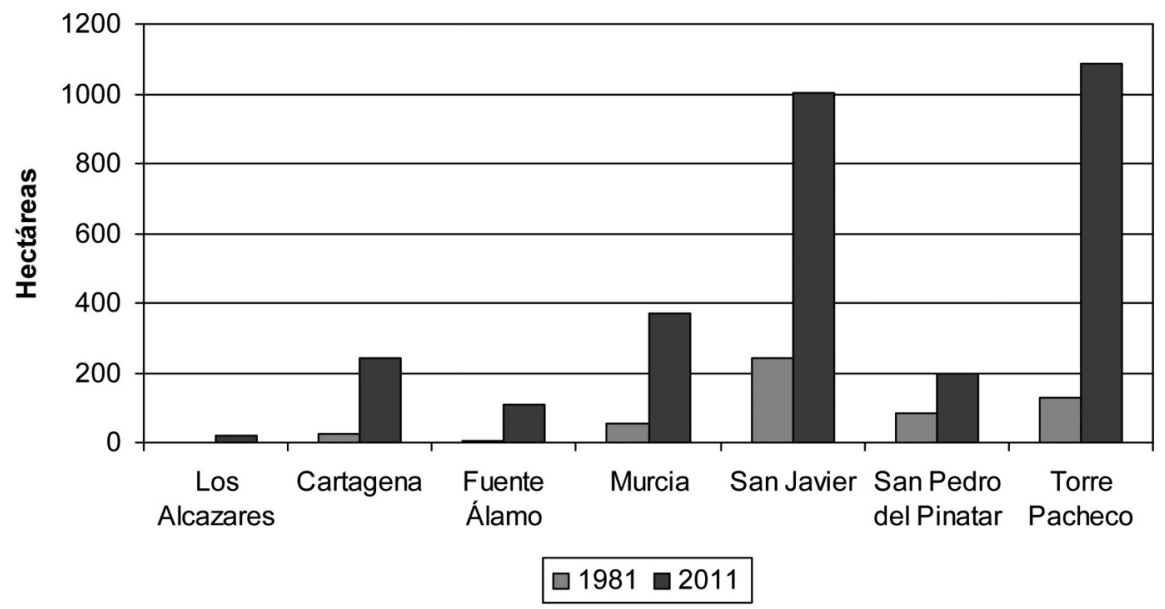

Fuente: Elaboración propia mediante digitalización

\section{Transformación paisajística en la comarca}

Como consecuencia de la alta proliferación de invernaderos, en la comarca analizada se ha producido una importante transformación paisajística. Hasta finales de los años 70 y principios de los 80 , la agricultura en la Comarca se caracterizaba por ser de carácter tradicional, en su mayor parte de secano, siendo el regadío existente sustentado por los aportes de acuíferos extraídos mediante pozos. La agricultura tecnificada bajo invernaderos llegó a finales de los años 70 e inicios de los 80 . En la figura 6 se puede observar cuál era el estado de los cultivos en los años 50 y 80 del siglo pasado, en los alrededores del Cabezo Gordo (municipio de Torre Pacheco).

En la actualidad, una gran parte de la superficie comarcal (3.032 ha) está ocupada por estas estructuras. En la figura 7 se muestra la distribución general de los invernaderos en la comarca en los años 1981 y 2011, y en estas imágenes se puede apreciar la importante transformación del espacio.

En 1981 los invernaderos aun no suponían un agente transformador de relevancia. Empezaban a desarrollarse, de forma aislada, al Norte del área de estudio, pero sin presentar una clara zonificación y densidad. La dicotomía secano-regadío propia de la Región de Murcia aun estaba latente en esta fecha, con una alternancia de regadío en invernaderos y cultivos al aire libre. Es en la imagen correspondiente a 2011, donde ya se puede apreciar el notable cambio en el paisaje. 
FIGURA 6:

PAISAJE AGRARIO EN LOS ALREDEDORES DEL CABEZO GORDO
(TORRE PACHECO)
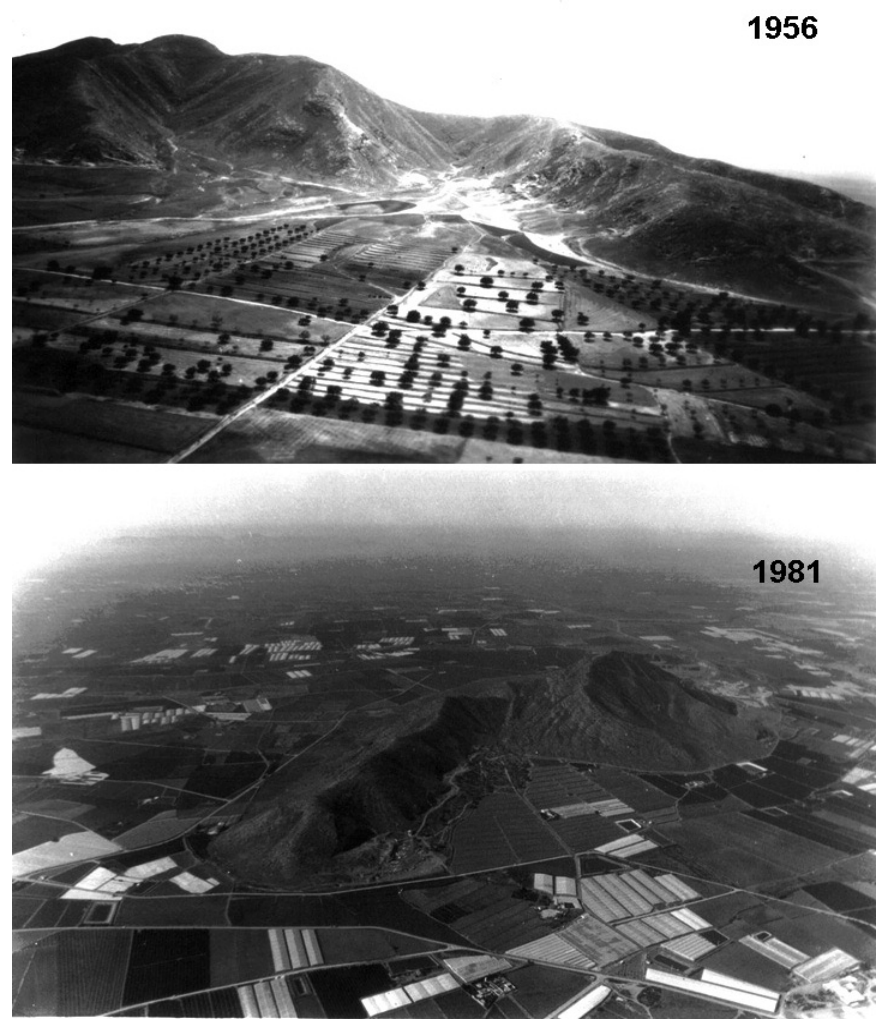

Fuente: Academia General del Aire

En el año 2011, se muestra casi un completo dominio de los invernaderos sobre el terreno. Se aprecia una clara zonificación en los municipios localizados al Norte de la comarca, dando lugar a una heterogeneidad del paisaje. Casi un tercio de la superficie del suelo se encuentra ocupada por invernaderos y el paisaje se presenta altamente modificado, pasando a formar parte del paisaje actual del Sureste español, colonizado por los cultivos bajo plástico. El paisaje tradicional, compartimentado por los distintos tipos de cultivos, sus diferentes teselas de cubiertas vegetales, con diversas tonalidades de marrones y verdes, ha quedado sustituida por la visión de una capa continua de plásticos. 


\section{FIGURA 7:}

INVERNADEROS EN LA CCMM. AÑOS 1981 Y 2011

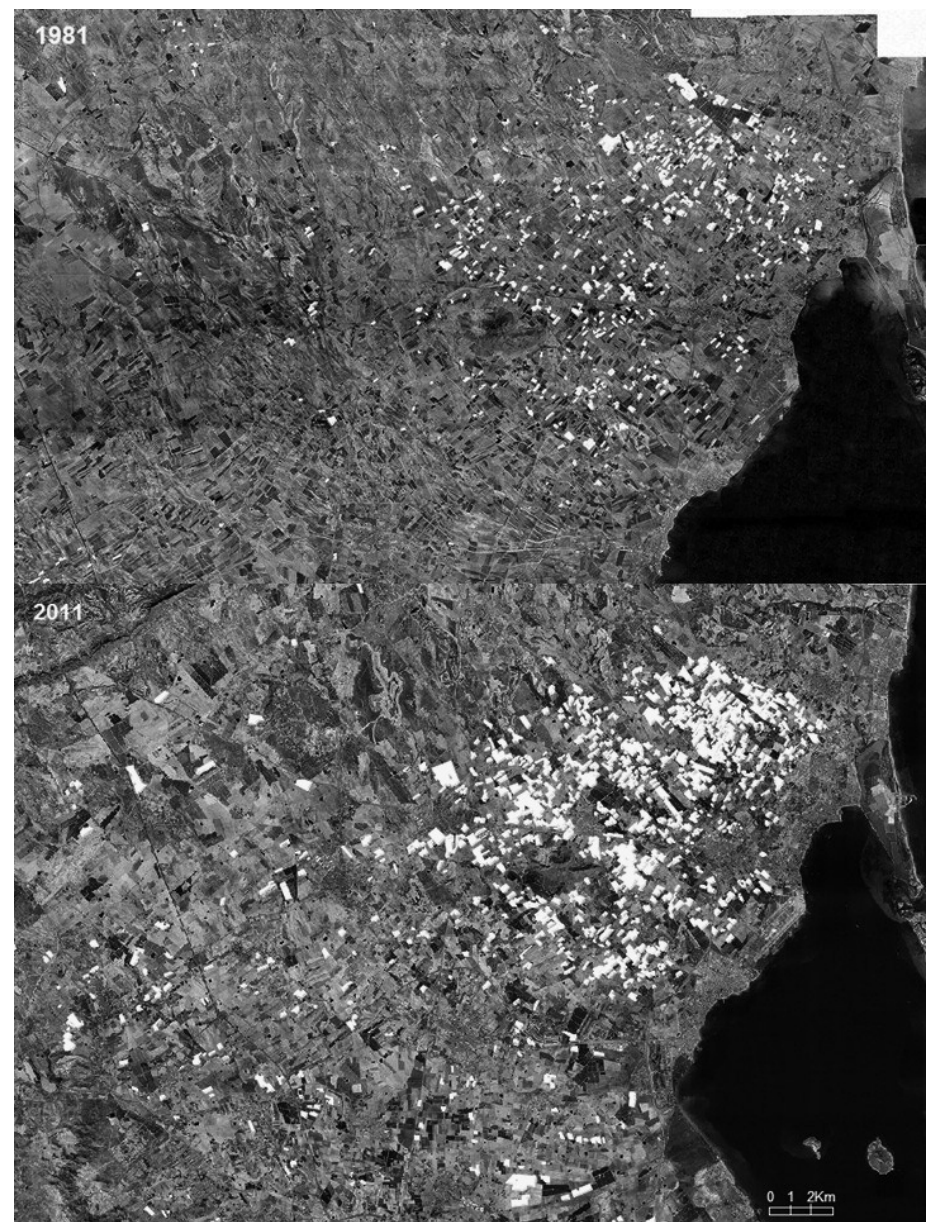

Fuente: Elaboración propia mediante digitalización, sobre la ortofoto de Cartomur.

La implantación de los invernaderos en la Comarca no se ha producido de forma homogénea. A fecha de 2011, los municipios que se han consolidado como los de mayor superficie de cultivos de invernadero son Torre Pacheco y San Javier. Esto supone que, de las 18.940 ha que tiene el primer municipio, 1.086 ha de su suelo están ocupadas por invernaderos. Mientras que, de las 7.510 ha de superficie que posee el municipio de San Javier, 1.002 ha se en-

Estudios Geográficos, Vol. LXXVI, 279, pp. 473-498, julio-diciembre 2015 ISSN: 0014-1496, eISSN: 1988-8546, doi: 10.3989/estgeogr.201517 
cuentran invadidas por estas instalaciones. La evolución que han sufrido estos municipios ha sido vertiginosa, sobre todo en el caso de San Javier, si se tiene en cuenta su pequeña superficie. El municipio que menos ha visto modificado su paisaje, por causa de los invernaderos, ha sido Los Alcázares. En esta localidad, de escasa superficie, y que cuenta con ayuntamiento desde 1983, la actividad económica se ha orientado más hacia el turismo de segunda residencia, de forma que los invernaderos son muy escasos. No obstante, la evolución con el paso de los años, se ha producido casi al mismo ritmo que en los dos casos anteriores, puesto que ha multiplicado por diez su superficie ocupada, aunque partía de un valor más pequeño.

Es de mencionar como no todos los municipios analizados han aumentado la superficie de invernaderos en el periodo 1981-2011 (tabla 2). Son de citar San Javier y San Pedro del Pinatar que la han visto notablemente disminuida, sin duda, por efecto del incremento de la superficie urbanizada en los últimos años, en detrimento de las superficies de cultivos, entre las que se encuentran los invernaderos.

TABLA 2:

SUPERFICIE (\%) DE CULTIVOS EN INVERNADEROS EN LOS DISTINTOS MUNICIPIOS ESTUDIADOS (1981-2011)

\begin{tabular}{lcc}
\multicolumn{1}{c}{ Municipio } & 1981 (\%) & 2011 (\%) \\
\hline Los Alcázares & 0 & 1 \\
Cartagena & 4 & 8 \\
Fuente Álamo & 1 & 4 \\
Murcia & 11 & 12 \\
San Javier & 45 & 33 \\
San Pedro del Pinatar & 15 & 7 \\
Torre Pacheco & 24 & 36 \\
\hline
\end{tabular}

Fuente: Elaboración propia a partir de datos del CREM. 


\section{Efectos medioambientales de los invernaderos}

La agricultura intensiva de invernaderos, a pesar de todas las ventajas socio-económicas que supone, ocasiona, sin embargo, toda una serie de impactos medioambientales muy importantes. Ha sido considerada como un modelo de explotación de los recursos naturales, muy similar a la de la industria, de hecho ha aparecido el concepto de «agricultura industrial» (Massaro et al. 1999), debido al alto grado de cambios y equipamientos que exige en el territorio, así como a los elevados valores de insumos y niveles productivos que la caracterizan, propios de regiones industrializadas.

Antón Vallejo (2004) califica a este tipo de agricultura como un brutal atentado al paisaje, además de conllevar graves riesgos de contaminación residual, y Gómez (2011), cita hasta una decena de impactos derivados de estas actuaciones sobre el medio, destacando la contaminación de la atmósfera, los acuíferos y el riesgo de incendios, así como la posible contaminación en las aguas del litoral por la concentración masiva de residuos orgánicos agrícolas. Por otra parte, Matarán Ruíz (2006) menciona que los invernaderos constituyen la actividad agrícola más impactante para el medio ambiente, ya que son sistemas altamente productivos que necesitan grandes cantidades de recursos, producen numerosas y mayores cosechas, pero también grandes cantidades de residuos.

Los principales impactos medioambientales de los invernaderos, y con incidencia en la comarca, se pueden resumir en los siguientes:

\section{a) Aumento de los recursos que consumen}

Sin duda, el recurso más importante consumido es el agua, pese a que estas instalaciones se han diseñado como estructuras de alta eficiencia, disminuyendo la evapotranspiración en un 50\% (López Gálvez y Salinas Andújar, 1998). Es necesario disponer de agua en cantidad suficiente para toda la superficie de invernaderos existente, por lo que en ocasiones se recurre a aguas subterráneas provocando la sobreexplotación y salinización de los acuíferos. Según el OSE (2006) en la Región de Murcia, recientemente se ha experimentado un incremento muy importante de las tierras de regadío en una zona con escasos recursos hídricos y graves problemas de sobreexplotación de acuíferos. En la actualidad, muchas de las nuevas tecnologías que se están comenzando a usar tratan de reducir el consumo de agua, como por ejemplo: los cultivos hidropónicos (García Lozano y Urrestarazu Gavilán, 1999), los siste- 
mas de riego automático (Fernández Fernández, 2003), los sistemas de control climático (Montero et al., 1998; García Lozano y Escobar Jiménez, 2001) y los sistemas de recogida del agua de lluvia; pero no todas ellas se están aplicando en la comarca del CCMM.

b) Aumento de los residuos que generan (líquidos, sólidos y gaseosos)

Los invernaderos son los mayores consumidores de pesticidas y fertilizantes, aunque la eficiencia de estas plantaciones es más elevada. Es evidente que si el consumo es mucho mayor, el vertido también lo será.

La mayor parte de los «residuos líquidos» provienen de los sobrantes del regadío, de la utilización de dosis excesivas y del lavado de los residuos sólidos gestionados de manera inadecuada (Olea, 1997). Atendiendo al lavado de residuos orgánicos, también existe un grave riesgo de contaminación tanto por fertilizantes como por pesticidas, que están muy presentes en esta fracción residual. En este caso, el riesgo para la salud de las personas se debe a que, en ocasiones, estos residuos contaminados son utilizados para alimentar al ganado (Fernández García, 1998). La producción media anual de sobrantes de regadío en un invernadero es de 0,3 toneladas por hectárea, incluyendo agua, fertilizantes y pesticidas, aunque la cantidad total podría llegar a alcanzar las 2,2 toneladas en algunos casos (Naredo, 1995; Antón Vallejo, 2004). Considerando el valor más bajo de $0,3 \mathrm{Tm} / \mathrm{ha}$ el balance de residuos líquidos procedente de los invernaderos $(0,3 \mathrm{Tm} / \mathrm{ha} * 3031,81$ ha de invernaderos en la CCMM), ascendería a $909.543 \mathrm{Tm}$.

En la comarca analizada hay un problema añadido, ya que gran parte de estos fertilizantes van a parar al Mar Menor. Los vertidos que provienen de la actividad agrícola de regadío intensivo asociada al lixiviado de cantidades crecientes de herbicidas, fertilizantes, fungicidas, etc., llegan a la laguna a través de los drenajes de las ramblas que desembocan en ella, principalmente por la rambla del Albujón, que recolecta gran parte de las aguas de escorrentía del Campo de Cartagena. En la actualidad esta rambla mantiene un flujo regular de agua debido a los cambios en las prácticas agrícolas (Pérez Ruzafa y Marcos, 2005). Los fertilizantes, sobre todo nitratos y compuestos de fósforo, suponen un riesgo importante tanto para la salud humana como para el medio natural, donde pueden desencadenar procesos de eutrofización. El aumento de la superficie de regadío ha generado un notable aumento de la entrada de nitrógeno y fósforo de origen agrícola al Mar Menor en las últimas décadas, hasta situarse en la actualidad en unos valores medios anuales en torno a las 
2.000 toneladas anuales en el caso del nitrógeno y 60 toneladas anuales en el caso del fósforo, cifras muy importantes si se considera el volumen de la laguna, su grado de confinamiento y el origen oligotrófico de sus aguas (Martínez Fernández y Esteve Selma, 2005). Por otra parte, los nitratos de los fertilizantes disueltos en el agua se han convertido en alimento para algunas especies de medusas (Cotylorhiza tuberculata y Rhizostoma pulmo), lo que les ha llevado a instalarse y a reproducirse con gran proliferación en el Mar Menor. En algunos años (verano de 1997) se han alcanzado cifras de hasta $40 \mathrm{mi}-$ llones de individuos en toda la laguna, con una densidad de 12 individuos por $100 \mathrm{~m}^{3}$ (Pérez Ruzafa y Marcos, 2005); precisamente cuando la actividad turística del Mar Menor y su entorno es máxima. De modo que los efectos negativos de dicha proliferación sobre la calidad del baño, las actividades recreativas y la imagen turística del Mar Menor son considerables.

Respecto a la producción de «residuos sólidos», esta tipología de agricultura industrial produce hasta diez veces más residuos que el resto de agriculturas (Alcalde, 2003). La biomasa y el plástico de cobertura representan el mayor porcentaje de producción de residuos.

Los residuos orgánicos suponen la principal fracción de residuos de invernaderos. Siguiendo a Alcalde (2003) y Fernández García (1998), la cantidad de residuos producida y el momento de producción dependerá de las características del cultivo y, más concretamente, de las peculiaridades de las variedades, realización de podas, estado de lignificación, marco de plantación o fechas de arranque. Pero el volumen total puede ser mucho mayor teniendo en cuenta los productos que se retiran cuando los precios bajan (Grupo Ecologista Mediterráneo, 1999) o cuando se produce alguna de las plagas que azotan a estos cultivos de forma generalizada, a causa de la saturación territorial y de la falta de medidas higiénicas (Matarán Ruiz, 2005).

La cantidad global de residuos plásticos de cubierta oscila entre 900 y $1.100 \mathrm{~kg} / \mathrm{ha}$ (Antón Vallejo, 2004). Esta ingente cantidad de residuos plásticos, que resultan altamente perjudiciales para la salud y el medio ambiente, precisan de un correcto tratamiento, que no siempre se lleva a cabo (Prieto Prieto, 2009). Los residuos, la mayoría de las veces no son trasladados a los centros de incineración y reciclado, se quedan junto a los caminos o son quemados sin control alguno, a pesar de que en la actualidad en la CCMM hay tres plantas de reciclado de plásticos agrícolas.

De entre los contaminantes "gaseosos», una de las principales fuentes son los pesticidas desprendidos durante el proceso de aplicación. Se ha llegado a medir la contaminación por estas sustancias a distancias mayores de los $60 \mathrm{~km}$ (Alcalde, 2003), aunque la peor parte se la llevan los trabajadores de los inver- 
naderos, cuya salud está expuesta a importantes riesgos, tal y como se ha recogido en varios estudios (García et al., 2004; Olea, 1997). En la comarca, en determinadas épocas del año, es fácil observar nubes de estos contaminantes.

\section{c) Aumento del riesgo de erosión e inundaciones}

Al ser la CCMM prácticamente una llanura, los posibles cambios topográficos para la construcción de invernaderos que pudieran generar riesgos de erosión aquí no son muy importantes. Por el contrario, lo que sí se ha incrementado con la construcción de los invernaderos es el riesgo de inundación (Camacho Olmedo, 1996; Fernández Marín et al., 1998; Alcalde, 2000). Las cubiertas plásticas son impermeables, por lo que ejercen un efecto de sellado de los suelos, impidiendo la infiltración del agua y favoreciendo las escorrentías. Por otro lado, la deficiente gestión de los residuos supone la acumulación ilegal de éstos en las ramblas y otros cauces, reduciendo su capacidad y actuando de presas (Alcalde, 2000); al tiempo que algunas ramblas han sido ocupadas por cultivos de invernaderos (Belmonte Serrato et al., 2011). La superficie ocupada por invernaderos, sumada a las superficies artificiales, que se han visto incrementadas en los últimos años, especialmente en el litoral de la Región de Murcia (OSE, 2006), y en particular en la comarca aquí analizada (Romero Díaz et al., 2011b) hace que el riesgo de inundación se haya visto incrementado de forma muy notable y que sufra de forma recurrente los efectos de las avenidas (Romero Díaz et al., 2010).

\section{d) Reducción de la biodiversidad}

Mientras que la mayoría de sistemas agrarios interaccionan con el ambiente circundante, los invernaderos están diseñados precisamente para estar aislados del medio. Por lo tanto, espacios que eran parte del ecosistema ahora se han transformado en superficies agro-industriales aisladas del sistema de relaciones ecosistémico. Además, la contaminación producida por el vertido de residuos sólidos, líquidos y gaseosos supone también un serio peligro sobre la biodiversidad (Matarán Ruíz, 2006).

\section{e) Otros efectos}

Alteraciones olfativas en el medio, como consecuencia de la utilización de abonos orgánicos y fumigaciones; y alteraciones auditivas del medio, como 
ruido de motores de extracción de aguas, diversa maquinaria agrícola y de recolección, constituyen también otros efectos negativos sobre las poblaciones aún por evaluar.

\section{CONCLUSIONES}

En la actualidad la Región de Murcia es uno de los territorios peninsulares con mayor implantación de la agricultura intensiva bajo invernaderos, sólo superada por Andalucía y Canarias.

Se ha constatado el incremento de superficie ocupada por invernaderos en la Comarca del Campo de Cartagena-Mar Menor, desde los años ochenta del Siglo Xx, en especial debido a la llegada de las aguas del trasvase Tajo-Segura.

Son destacables las diferencias en la superficies dedicadas al cultivo mediante invernaderos según datos oficiales (1.495 ha) y los obtenidos por digitalización (3.032 ha). La razón puede estar, por un lado, en que muchos de los invernaderos no están declarados como tales y, por otro, que mediante digitalización se contabiliza una superficie un poco más amplia que la estrictamente del invernadero.

Aunque es evidente que la agricultura intensiva bajo invernaderos tiene aspectos socioeconómicos muy positivos (Valera Martínez et al., 2014), se ponen de manifiesto otros aspectos muy negativos desde un punto de vista medioambiental.

La aparición de los invernaderos ha supuesto un avance en cuanto a rendimiento agrícola se refiere, aunque constituyen una potente herramienta de impacto paisajístico. La modernización de la agricultura en la Comarca del CCMM ha generado la creación de una verdadera barrera de plástico, transformando el paisaje completamente. Los invernaderos se han consolidado como estructuras complejas que han traído consigo la pérdida masiva de terreno natural, no solo el que queda bajo las instalaciones, sino también, el que queda entre un invernadero y otro.

La concentración masiva de invernaderos en los últimos años ha ocasionado la generación de grandes cantidades de residuos líquidos y sólidos, que se unen a los problemas de impacto resultantes de su implantación. El vertido de fertilizantes y pesticidas de manera superficial y subsuperficial a la laguna del Mar Menor ha ocasionado un impacto medioambiental muy negativo con los consiguientes efectos para el turismo. Por otra parte, la elevada cantidad de residuos ha generado un descontrol administrativo en cuanto a la eliminación de plásticos y la utilización racional y sostenible de pesticidas y herbicidas. 
Otro de los efectos que generan los invernaderos es el incremento de las escorrentías. Las cubiertas de los invernaderos, en la mayoría de los casos, son impermeables, por lo que aumentan los caudales superficiales (en especial en épocas de lluvias intensas), favoreciendo las avenidas e inundaciones.

No resulta fácil estimar cuándo tendrá lugar un cambio en este tipo de agricultura para que resulte más sostenible y respetuosa con el medio. Afortunadamente, se ha comenzado a tomar conciencia sobre este tema y son varios los estudios que se han realizado sobre el impacto ambiental de la nueva agricultura y sus posibles efectos y soluciones (Bellarby et al., 2008). Ya se han implantado algunas bases, como son la instalación plantas de reciclado y tratado de residuos, solo falta concienciar a la población para seguir las directrices adecuadas. No obstante, la transformación del paisaje que este tipo de agricultura ha producido es posible que no cambie, debido a los importantes beneficios que de ella se obtienen.

Fecha de recepción: 5 de agosto de 2014.

Fecha de aceptación: 8 de Julio de 2015.

\section{BiBLIOGRAFÍA}

Alcalde, F. (2000): "Riesgo de inundaciones inducido por la agricultura intensiva bajo plástico", en: V Congreso Nacional de Medio Ambiente. Madrid. http://issuu.com/pegaozillo/docs/vi_conama (Verificada: 017/09/2015).

Alcalde, F. (2003): "Residuos de las actividades agrícolas", presente en la Documentación del Máster en Consultoría Medioambiental. Granada, Grupo Najarra Lencom, (sin publicar).

Antón Vallejo, M. A. (2004): "El invernadero mediterráneo", en: Utilización del Análisis del ciclo de vida en la evaluación del impacto ambiental del cultivo bajo invernadero mediterráneo. Barcelona, Universidad Politécnica de Cataluña, pp. 23-42.

Bellarby, J.; Foereid, B.; Hastings, A. and Smith, P. (2008): Cool Farming: climate impacts of agriculture and mitigation potential, Amsterdam, Greenpeace International, $43 \mathrm{pp}$.

Belmonte Serrato, F; Romero Díaz, A.; Rupérez Tirado, E. y Moreno Brotóns, J. (2011): "El impacto de la agricultura intensiva en el uso turístico de las playas de Marina de Cope (Murcia)". Cuadernos de Turismo, 27, pp. 23-38.

Camacho Olmedo, M. T. (1996): Cartografía de los paisajes erosivos de la Sierra de la Contraviesa (provincias de Granada y Almería). Granada, Universidad de Granada.

CARTOMUR. Enlace del Servicio de Cartografía de la Región de Murcia. Disponible en http://www.cartomur.es (Verificada: 01/09/2015). 
Castilla, N. (1998): "Condiciones ambientales en invernaderos no climatizados". Tecnología de invernaderos, II, pp. 163-177.

Cobos, J. J. y López, J. C. (1998): "Filmes plásticos como material de cubierta de invernadero". Tecnología de invernaderos, II, pp. 143-160.

Conesa García, C. (1990): El Campo de Cartagena. Clima e hidrología de un medio semiárido. Murcia, Universidad de Murcia, Ayuntamiento de Cartagena y Comunidad de Regantes del Campo de Cartagena, 450 pp.

Costa Mas, J. y Canales Martínez, G. (1980): "El cultivo en invernadero y la comercialización agraria en Orihuela y Campo de Cartagena". Cuadernos de Geografía, 27, pp. 173-202.

Cotec (2009): "Invernaderos de plástico", en: Documentos Cotec sobre oportunidades tecnológicas, 26, 102 pp. Madrid, Fundación Cotec para la información tecnológica.

CREM (varios años): Anuarios estadísticos de la Región de Murcia. Centro Regional de Estadística de Murcia. Disponible en http://www.carm.es/econet/ (Verificada: 01/09/2015).

Espejo Marín, C. (2008): "La electricidad térmica en la Región de Murcia”, en: Estudios sobre desarrollo regional. Murcia, Servicio de Publicaciones de la Universidad de Murcia, pp. 95-129.

FAO (2013): Good Agricultural Practices for greenhouse vegetable crops. Roma, Principles for Mediterranean climate areas, FAO, 640 pp.

FAUGT (2008): Medio Ambiente y trabajos en invernaderos. Madrid, Ministerio de Medio Ambiente y Medio Rural y Marino, 168 pp.

Fernández Fernández, M. D. (2003): "Programación del riego mediante parámetros climáticos: Cultivos hortícolas bajo invernaderos en el sudeste español". Riegos y drenajes del S. XXI, Número Especial Almería, pp. 44-49.

Fernández García, F. (1998): "Criterios específicos en la elección de los sistemas para la recogida de residuos agrícolas procedentes de la agricultura intensiva”, en: Encuentro Medioambiental Almeriense. Almería, Instituto de Estudios Almerienses, CD-ROM (D:/DOCUMENT/DOCUMEN/g11/d11208/d11208.htm).

Fernández Marín, E.; Aguilera Aguilera, P.; Castro Nogueira, H. y López Carrique, E. (1998): "Residuos sólidos agrícolas. Impactos Ambientales", en: Encuentro Medioambiental Almeriense. Almería, Instituto de Estudios Almerienses, CD-ROM (D:/DOCUMENT/DOCUMEN/g11/d11204/d4.htm1120).

García Lorca, A. (1999): "Tendencias y transformaciones en la agricultura intensiva almeriense", Nimbus, 4, pp. 109-128.

García Lozano, M. y Urrestarazu Gavilán, M. (1999): Recirculación de la disolución nutritiva en las condiciones de los invernaderos de la Europa del sur. Granada, Gabinete Técnico de la Caja Rural de Granada.

García Lozano, M. y Escobar Jiménez, I. (2001): Diez años de experiencias en cultivos sin suelo. Granada, Gabinete Técnico de la Caja Rural de Granada.

García, A. M.; Gadea, R.; Muñoz, M. I.; Cano, I. y González, F. (2004): Diagnóstico de 
salud laboral en invernaderos agrícolas de Almería. Madrid, Instituto Sindical de Trabajo, Ambiente y Salud (ISTAS), Comisiones Obreras.

Gil Meseguer, E. y Gómez Espín, J. M. (2011): "Cultivos bajo cubierta en el Sureste de España”. Papeles de Geografía, 53-54, pp. 155-170.

Gil Meseguer, E. y Gómez Espín J. M. (2014): "Los paisajes rurales del Campo de Cartagena-Mar Menor. Del riego itinerante a la factoría bajo cubierta", en F. Molinero (coord.): Atlas de los paisajes agrarios de España. Madrid, MAGRAMA, vol. II, pp. 543-552.

Gómez, J. E. (2011): "Waste magazine". Obtenido May 2011, de Contaminación e Invernaderos: http://waste.ideal.es/invernaderos.htm (Verificada: 01/09/2015).

González Ortiz, J. L. (2007): "Comarca de Cartagena - Mar Menor", en A. Romero Díaz y F. Alonso Sarria (coords.): Atlas Global de la Región de Murcia. Murcia, La Verdad - CMM S.A., pp. 478-489.

Grupo Ecologista Mediterráneo (1999): Los residuos agrícolas en el Poniente Almeriense. Almería.

Hernández Porcel, M. A. (2005): "La agricultura intensiva en el contexto territorial del litoral de Almería”, en A. Viciana Martínez Lage y A. Galán Pedregosa (coords.): El Litoral de Almería, caracterización, Ordenación y Gestión de un espacio geográfico. Almería, Instituto de Estudios Almerienses, pp. 53-71.

INE (2012): Instituto Nacional de Estadística. http://www.ine.es/ (Verificada: 01/09/2015).

López Gálvez, J. y Salinas Andújar, J. A. (1998): "Efectos ambientales del sistema de cultivo forzado", en: Encuentro Medioambiental Almeriense. Almería, Instituto de Estudios Almerienses. CD-ROM (D:/DOCUMENT/DOCUMEN/g02/d02201/ d02201.htm).

MAAMA (2002): Encuesta sobre superficies y rendimientos de cultivos en España (ESYRCE). Madrid, Ministerio de Agricultura, Alimentación y Medio Ambiente, $195 \mathrm{pp}$.

MAAMA (2013): Encuesta sobre superficies y rendimientos de cultivos en España (ESYRCE). Madrid, Ministerio de Agricultura, Alimentación y Medio Ambiente, $166 \mathrm{pp}$.

Martínez Fernández, J. y Esteve Selma, M. A. (2005): "Estimación de la contaminación agrícola en el Mar Menor mediante un modelo dinámico". Mediterránea. Serie de Estudios Biológicos, 18, pp. 8-35

Martínez Hernández, C.; Cánovas García, F; Alonso Sarria, F.; Romero Díaz, A. y Belmonte Serrato, F. (2013): "Cartografía de áreas agrícolas abandonadas mediante técnicas de SIG y fotointerpretación. Comarcas de la huerta y Campo de Murcia y Alto Guadalentín". Espacios insulares y de frontera, una visión geográfica. Palma de Mallorca, Universitat de les Illes Balears, pp. 393-403.

Martínez Menchón, M. y Senent Alonso, M. (2007): "El agua en el Campo de Cartagena". Revista Murciana de Antropología, 14, pp. 47-61.

Massaro, A.; Pazos, P.; Gómez, J. L.; Olea, M. F. y Rivas Olea, N. (1999): "Impacto de la agricultura intensiva en el Sudeste español: Transformación urbana del medio 
ambiente", en: Minería, Industria y Medio Ambiente en la Cuenca Mediterránea. http://www.asociacionbuxus.org/administracion/archivos/Invernaderos.pdf (Verificada: 017/09/2015).

Matarán Ruiz, A. (2005): "La valoración ambiental-territorial de las agriculturas de regadío en el litoral Mediterráneo: el caso de Granada", Tesis Doctoral, Universidad de Granada.

Matarán Ruíz, A. (2006): "Análisis de los impactos ambientales producidos por los invernaderos". Residuos, 94, pp. 66-77.

Montero, J. I.; Antón, A. y Muñoz P. (1998): "Modificaciones en las estructuras e instalaciones de invernaderos orientadas a la reducción del impacto ambiental", en: Encuentro Medioambiental Almeriense. Almería, Instituto de Estudios Almerienses, CD-ROM (D:/DOCUMENT/DOCUMEN/g02/d02101/d02101.htm).

Morales Gil, A. (1997): Aspectos geográficos de la horticultura de ciclo manipulado en España. Alicante, Universidad de Alicante, Secretariado de publicaciones, 168 pp.

Naredo, J. M. (1995): "Territorio, Medio Ambiente y Agricultura de Invernadero", en J. López Gálvez, y J. R. Díaz Álvarez: Actas del I Simposium Iberoamericano sobre "Aplicación de los plásticos en las tecnologías agrarias". El Ejido, Almería, CEPLA y FIAPA, pp. 463-470.

Olea, N. (1997): "Health effects of pesticides", en J. M. Shuman (ed.): The Int. Conf. on Regulatory Issues in Crop Protection and Their Implications for the Food Supply". Boston, Editorial Boston, pp, 38-40

OSE (2006): Cambios de Ocupación del Suelo en España: Implicaciones para la Sostenibilidad. Observatorio de la Sostenibilidad en España. http://www.upv.es/contenidos/ CAMUNISO/info/U0637086.PDF. (Verificada: 01/09/2015).

Pérez Parra, J.; López, J. C. y Fernández, M. D. (2002): "La agricultura del Sureste: situación actual y tendencias de las estructuras de producción en la horticultura almeriense", en J. M. García Álvarez-Coque (ed): La Agricultura Mediterránea en el siglo XXI. Mediterráneo Económico, 2, pp. 262-282.

Pérez Ruzafa, A. y Marcos, C. (2005): "Pressures on Mediterranean coastal loagoons as a consequence of human activities", in C. A. Fletcher and S. Spencer: Flooding and environmental challenges for Venice and its lagoon: State of knowledge. Cambridge, University Press, pp. 545-556.

Prieto Prieto, J. A. (2009): "Agricultura y ganadería en el Campo de Cartagena como modelo de los cambios recientes que ha experimentado el sector primario de la Región de Murcia", en: $3^{a}$ Edición de premios a la elaboración de materiales curriculares sobre la identidad de la Región de Murcia. Murcia, Consejería de Educación, Formación y Empleo, pp. 11-62.

Román Cervantes, C. (2007): "El control del agua: tecnología y sistemas de extracción hidráulica en la comarca del Campo de Cartagena, 1880-1980". Revista Murciana de Antropología, 14, pp. 127-141.

Romero Díaz, A.; Belmonte Serrato, F; Ruiz Sinoga, J. D. y Pérez Correas, J. D. (2010): "Episodio de lluvias torrenciales del 27-28 de septiembre de 2009. Las inundacio- 
nes del campo de Cartagena (Murcia)", presentado a las II Jornades de Meteorología i Climatología de la Mediterrània Occidental. Valencia. Associació Catalana de Meteorología. Presentación en Poster.

Romero Díaz, A. y Belmonte Serrato, F. (2011a): "El Campo de Cartagena una visión global", en J. Hernández Bastida (coord.): Recorridos por el Campo de Cartagena. Control de la degradación y uso sostenible del suelo. Murcia, Instituto Mediterráneo del Agua, pp. 17-48.

Romero Díaz, A.; Belmonte Serrato; Do Campo Calvo, A. M. y Ruíz Sinoga, J. D. (2011b): "Consecuencias del sellado de los suelos en el Campo de Cartagena (Murcia)", en V. Gonzálvez Pérez y J. A. Marco Molina (eds.): Urbanismo expansivo de la utopía a la realidad. Alicante, Universidad de Alicante, pp. 605-616.

Sigrimis, N.; Cavallini, A.; Incrocci, L.; Montero, J. I.; Perez-Parra, J. y Kafka, A. (2009): Data-collection of Existing Data on Protected Crop Systems (Greenhouses and Crops Grown Under Cover) in Southern European EU Member States. Athens (Greece), European Food Safety Authority.

Tesi, R. (1972): Moderne tecniche di protezione in orticoltura, floricoltura e frutticoltura. Bologna, Edagricole, 277 pp.

Tolón Becerra, A. y Lastra Bravo, X. (2010): "La agricultura intensiva del Poniente Almeriense. Diagnóstico e instrumentos de gestión ambiental". Revista electrónica de Medio Ambiente, 22 pp. Universidad Complutense de Madrid.

Valera Martínez, D. L.; Belmonte Ureña, L. J.; Molina Aiz, F. D. y López Martínez, A. (2014): Los invernaderos de Almería. Análisis de su tecnología y rentabilidad. Almería, Cajamar Caja rural, 504 pp. Colección Serie Economía, 21.

\section{RESUMEN}

El cultivo mediante invernaderos ha supuesto una de las mayores transformaciones agrícolas en el mundo, pero en especial en el sureste español. El objetivo general de este estudio ha sido analizar la transformación del paisaje y algunos de sus efectos medioambientales que, en la comarca de Cartagena-Mar Menor, ha tenido lugar en las últimas décadas, como consecuencia de la instalación masiva de invernaderos. Para ello se han evaluado las superficies ocupadas por invernaderos en varios años de referencia, mediante digitalización de ortofotos y análisis estadístico. Los resultados muestran un aumento creciente de la superficie de invernaderos, desde 1980, relacionado con el trasvase Tajo-Segura. Este tipo de agricultura intensiva tiene aspectos socioeconómicos muy positivos, pero se ponen de manifiesto otros aspectos negativos desde un punto de vista medioambiental: residuos generados (sólidos y líquidos), aumento de las escorrentías y por tanto de las inundaciones, o la posible contaminación del Mar Menor.

PAlabras Clave: Agricultura intensiva; transformación paisajística; invernaderos; impactos medio-ambientales; Sureste de España. 


\begin{abstract}
The culture by greenhouses has been one of the greatest agricultural transformations in the world, but especially in the southeastern Spanish. The main objective of this study was to analyze the transformation of the landscape and some of its environmental effects that, in the region of Cartagena-Mar Menor, have taken place in the recent decades as a result of the massive installation of greenhouses. We evaluated the areas occupied by greenhouses in some reference years, by digitizing orthophotos and statistical analysis. The results show a steady increase of the area occupied by greenhouses since 1980, related with the arrival of the waters from river Tajo to Segura. This type of intensive agriculture has very positive economic aspects, but show other negative from an environmental viewpoint: waste (solid and liquid), increasing the runoff and therefore flooding, or the possible contamination of the Mar Menor.
\end{abstract}

KEY WORDS: Intensive agriculture; landscape transformation; greenhouses; environmental impacts; Southeast Spain.

\title{
RÉSUMÉ
}

La culture en serre a été l'une des plus grandes transformations agricoles dans le monde, et particulièrement dans le sud-est espagnol. Lobjectif général de cette étude était d'analyser la transformation du paysage et certains de ses effets sur l'environnement dans la région de Cartagena-Mar Menor, transformation qui a eu lieu au cours des dernières décennies en raison de l'installation massive de serres. Cette étude a été pratiquée sur des zones occupées par des serres pendant plusieurs années par orthophotos numérisées suivie d'une analyse statistique.

Les résultats montrent une surface de plus en plus occupée par les serres depuis 1980, en raison de l'arrivée d'eau de la rivière Tajo à la Segura. Ce type d'agriculture intensive a des aspects économiques très positifs, mais d'un point de vue environnemental on relève aussi des aspects très négatifs: les déchets et autres résidus toxiques (solides et liquides), l'augmentation du ruissellement et donc des inondations, ou d'une possible contamination de la Mar Menor.

MoTS CLÉS: l'agriculture intensive; transformation du paysage; serres; impacts environnementaux; Espagne du Sud-Est. 